\title{
Vortex shedding from a circular cylinder near a moving ground
}

\author{
Takafumi Nishino, ${ }^{\text {a) }}$ Graham T. Roberts, ${ }^{\text {b) }}$ and Xin Zhang ${ }^{\text {c) }}$ \\ School of Engineering Sciences, University of Southampton, Southampton, SO17 1BJ, United Kingdom
}

(Received 28 June 2006; accepted 23 January 2007; published online 27 February 2007)

\begin{abstract}
The flow and force characteristics have been experimentally investigated of a circular cylinder with an aspect ratio of 8.33, with and without end-plates, placed near and parallel to a moving ground, on which substantially no boundary layer developed to interfere with the cylinder. Mean drag and lift measurements, surface oil flow visualization, and particle image velocimetry (PIV) measurements were carried out at two upper-subcritical Reynolds numbers of 0.4 and $1.0 \times 10^{5}$ (based on the cylinder diameter $d$ ) to investigate the mechanisms of the ground effect, i.e., the effect of the gap-to-diameter ratio $h / d$, where $h$ is the gap between the cylinder and the ground. For the cylinder with end-plates, on which the oil flow patterns were observed to be essentially two-dimensional, the drag rapidly decreased as $h / d$ decreased to less than 0.5 but became constant for $h / d$ of less than 0.35 , unlike that usually observed near a fixed ground. This critical drag behavior was found to be directly related to a global change in the near wake structure of the cylinder; as $h / d$ decreased the Kármán-type vortex shedding became intermittent at $h / d=0.4$, and then totally ceased and instead two nearly parallel shear layers were formed behind the cylinder at $h / d=0.3$ and below. For the cylinder without end-plates, however, no such critical change in drag was observed as the Kármán-type vortices were not generated in the near wake region at all $\mathrm{h} / \mathrm{d}$ investigated. Based on the experimental results obtained, further discussions are also given to the essential cause of the cessation of the Kármán vortex shedding in the ground effect. () 2007 American Institute of Physics. [DOI: 10.1063/1.2710273]
\end{abstract}

\section{INTRODUCTION}

Flow around a circular cylinder placed near a plane boundary has been the subject of a number of studies as it is of fundamental interest as well as of relevance to many engineering applications. In particular the onset and cessation of the Kármán-type vortex shedding observed in this flow configuration is of great interest as it may significantly affect various fluid-mechanical properties of practical importance, such as flow-induced forces, vibrations and noise, and the efficiencies of heat and mass transfer. In general the characteristics of this type of flow are governed not only by the Reynolds number Re but also by the "gap ratio," i.e., the ratio of the gap distance between the cylinder and the plane boundary, $h$, to the cylinder diameter $d$. However, the details of the effects of $h / d$, or "ground effect," are still far from being fully understood due to the existence of other influencing factors, such as the state of the boundary layer formed on the plane boundary, the aspect ratio of the cylinder, and the spanwise-end condition of the cylinder. The objective of the present study is to elucidate the mechanisms of the ground effect in more detail by wind tunnel experiments using: (i) a moving ground, on which substantially no boundary layer develops to interfere with the cylinder, whereas most of the earlier studies were conducted with a fixed ground as will be reviewed below, and (ii) a pair of end-plates, with and without which the influence of the spanwise-end condition of the

\footnotetext{
a)Electronic mail: nishino@soton.ac.uk

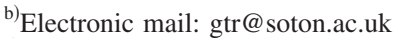

${ }^{c)}$ Electronic mail: xzhang@soton.ac.uk
}

cylinder in the ground effect is explicitly examined. The focus of this paper is mainly on the relation between the cessation of the Kármán vortex shedding and the drag reduction of the cylinder in the ground effect, and some discussions are also given to the cause itself of the cessation of the Kármán vortex shedding. But before that, a brief review of earlier experiments on this type of flow is given below.

As concerns the cessation of the Kármán-type vortex shedding in the ground effect, one of the earliest experiments was carried out by Taneda, ${ }^{1}$ who visualized the flow behind a circular cylinder towed through water close to a stationary wall at a very low Reynolds number of 170 (based on the cylinder diameter); the regular Kármán-type vortex shedding occurred at $h / d=0.6$, whereas only a weak single row of vortices was generated at $h / d=0.1$. On the other hand, for the force variation of the cylinder, Roshko et al. ${ }^{2}$ measured the time-averaged drag and lift coefficients of a circular cylinder placed near a fixed plane wall at an "upper-subcritical" Reynolds number of $2.0 \times 10^{4}$; the drag rapidly decreased and the lift increased as the cylinder came close to the wall, as reproduced later in Figs. 3 and 4, respectively [note that in the upper-subcritical flow regime $\left(2.0 \times 10^{4}<\operatorname{Re}<2.0\right.$ $\times 10^{5}$ ) the drag coefficient of a circular cylinder outside the ground effect shows an almost constant, high value of about 1.2 to 1.3 as the transition to turbulence takes place just after the flow separation from the cylinder surface, see, e.g., Ref. 3]. Bearman and Zdravkovich ${ }^{4}$ measured the pressure distribution around a circular cylinder also near a fixed ground in the upper-subcritical regime, and thereby explained the variations of the drag and lift coefficients of the cylinder in 
TABLE I. Summary of earlier experiments on the flow around a circular cylinder near a plane boundary.

\begin{tabular}{|c|c|c|c|c|c|}
\hline Authors & $\operatorname{Re}$ & $h / d$ & $\delta_{B} / d$ & $l / d$ & Key measurements \\
\hline Taneda $^{a}$ & 170 & $0.1-0.6$ & 0 & n.a. & $\mathrm{WFV}^{\mathrm{b}}$ \\
\hline Roshko et al. ${ }^{\mathrm{c}}$ & $2.0 \times 10^{4}$ & $0-6.0$ & 0.5 & n.a. & $C_{D}, C_{L}$ \\
\hline Bearman and Zdravkovich $^{\mathrm{d}}$ & $2.5 \times 10^{4}-4.8 \times 10^{4}$ & $0-3.5$ & 0.8 & 32 & $C_{p}, \mathrm{St}, \mathrm{SFV}^{\mathrm{e}}$ \\
\hline Buresti and Lanciotti ${ }^{\mathrm{f}}$ & $8.5 \times 10^{4}-3.0 \times 10^{5}$ & $0-2.5$ & 0.1 & 6.6 & St \\
\hline Angrilli et al. ${ }^{\mathrm{g}}$ & $2860-7640$ & $0.5-6.0$ & $<0.25$ & 9 & St \\
\hline Grass et al. ${ }^{\mathrm{h}}$ & $2000-4000$ & $0-2.0$ & $0.28-6.0$ & 20 & St, WFV ${ }^{\mathrm{b}}$ \\
\hline Zdravkovich $^{\mathrm{i}}$ & 3550 & $0.1-1.6$ & 0 & 11.8 & $\mathrm{WFV}^{\mathrm{b}}$ \\
\hline Zdravkovich $^{\mathrm{j}}$ & $4.8 \times 10^{4}-3.0 \times 10^{5}$ & $0-2.0$ & $0.52-0.97$ & $13.5,25$ & $C_{D}, C_{L}$ \\
\hline Hiwada et al. $^{\mathrm{k}}$ & $2.0 \times 10^{4}$ & $0-4.0$ & $0.23-2.82$ & 20 & $C_{D}, C_{L}, \mathrm{St}, \mathrm{SFV}^{\mathrm{e}}, \mathrm{WFV}^{\mathrm{b}}$ \\
\hline Taniguchi and Miyakoshi ${ }^{1}$ & $9.4 \times 10^{4}$ & $0-3.0$ & $0.34-1.05$ & 14 & $C_{D}, C_{L}, \mathrm{St}$ \\
\hline Lei et $a .^{\mathrm{m}}$ & $1.4 \times 10^{4}$ & $0-3.0$ & $0.14-2.89$ & 26 & $C_{D}, C_{L}, C_{L}, C_{p}, \mathrm{St}$ \\
\hline Price $e t a l .{ }^{\mathrm{n}}$ & $1200-4960$ & $0-2.0$ & 0.45 & 16.25 & St, WFV, ${ }^{\mathrm{b}}$ PIV \\
\hline Zdravkovich $^{\circ}$ & $2.5 \times 10^{5}$ & $0.02-0.5$ & 0 & 21 & $C_{D}, C_{L}$ \\
\hline Lin et al. ${ }^{\mathrm{p}}$ & 780 & $0-4.0$ & $0,0.86-1.41$ & 33.1 & St, WFV, ${ }^{\mathrm{b}}$ PIV \\
\hline Present study & $4.0 \times 10^{4}-1.0 \times 10^{5}$ & $0.05-2.0$ & 0 & 8.33 & $C_{D}, C_{L}, \mathrm{SFV},{ }^{\mathrm{e}} \mathrm{PIV}$ \\
\hline
\end{tabular}

${ }^{\mathrm{a}}$ Reference 1 .

${ }^{\mathrm{i}}$ Reference 9.

${ }^{\mathrm{b}}$ Wake flow visualization.

${ }^{\mathrm{j}}$ Reference 11.

${ }^{\mathrm{c}}$ Reference 2 .

${ }^{\mathrm{d}}$ Reference 4.

${ }^{\mathrm{e}}$ Surface flow visualization.

${ }^{\mathrm{f}}$ Reference 6.

${ }^{\mathrm{g}}$ Reference 7

${ }^{\text {h }}$ Reference 8 .

${ }^{\mathrm{k}}$ Reference 12

${ }^{\mathrm{I}}$ Reference 24.

${ }^{\mathrm{m}}$ Reference 13

${ }^{\mathrm{n}}$ Reference 5.

${ }^{\circ}$ Reference 14

${ }^{\mathrm{p}}$ Reference 10

the ground effect. They also measured velocity fluctuations in the near wake of the cylinder to study the effect of $h / d$ on the Strouhal number of the vortex shedding, St, and reported that the critical gap ratio $(h / d)_{\mathrm{c}}$, at which the spectral peak of the local velocity fluctuations disappeared, was $0.2-0.3$ (it should be noted, however, that the disappearance of the spectral peak of the local velocity fluctuations does not necessarily ensure the global cessation of the vortex shedding ${ }^{5}$ ). Similar measurements of local velocity fluctuations and thereby of $(h / d)_{c}$ were also carried out at higher and lower Reynolds numbers, ${ }^{6-8}$ whereas more details of the global cessation of the Kármán vortex shedding were later visualized but only at lower Reynolds numbers, ${ }^{5,9,10}$ as summarized in Table I.

Although the fundamental effects of $h / d$ on the flow and force characteristics have been successfully observed by the above authors, the relation between the cessation of the Kármán vortex shedding, and the significant drag reduction is still unclear largely due to the confusing effects of the boundary layer formed on the ground, and this is the main reason why a moving ground was employed in the present study as already mentioned before. As concerns the influence of the boundary layer, Zdravkovich ${ }^{11}$ reported that the rapid decrease in drag occurred as the gap was reduced to less than the thickness of the boundary layer, $\delta_{B}$, on a fixed ground, and concluded that the variation of the drag coefficient was dominated by $h / \delta_{B}$ rather than by the conventional gap ratio $h / d$. However, Hiwada et al. ${ }^{12}$ reported, for the case of $\delta_{B} / d=0.23$, that the decrease in drag started around $h / d$ $=0.5$, at which the cylinder was still outside the thin wall boundary layer and the regular vortex shedding was still observed. A further investigation was conducted for a wider range of $\delta_{B} / d$ by Lei et al., ${ }^{13}$ but no clear relation was observed between $\delta_{B},(h / d)_{\mathrm{c}}$ and the gap at which the decrease in drag occurred in their measurements. More recently, Zdravkovich ${ }^{14}$ measured the drag on a circular cylinder placed near a moving ground at a higher Reynolds number of $2.5 \times 10,{ }^{5}$ and reported that, in contrast to all the above investigations, the decrease in drag due to the decrease in $h / d$ did not occur. It was not clear, however, whether this was attributed to the nonexistence of the boundary layer on the moving ground, or the higher Reynolds number that seems to be within the critical flow regime rather than within the subcritical flow regime, ${ }^{3}$ or any other influencing factors.

In the light of these previous experiments, the present study investigates the flow around a circular cylinder, with and without end-plates, placed near a moving ground at two upper-subcritical Reynolds numbers of 0.4 and $1.0 \times 10^{5}$ (based on the cylinder diameter $d$ ). As already noted, the moving ground was employed with the aim to avoid the confusing effects of the boundary layer on the ground and thereby to reveal the essence of the ground effect. For the majority of the experiments the end-plates were attached to the cylinder to ensure the quasi-two-dimensionality of the flow, but for some experiments the plates were removed to show the influence of the spanwise-end condition of the cylinder in the ground effect. In the following, the details of the experiments will be described in Sec. II, and then the results will be presented in Sec. III. Further discussions will be given in Sec. IV, and finally a concluding summary will be given in Sec. V. 


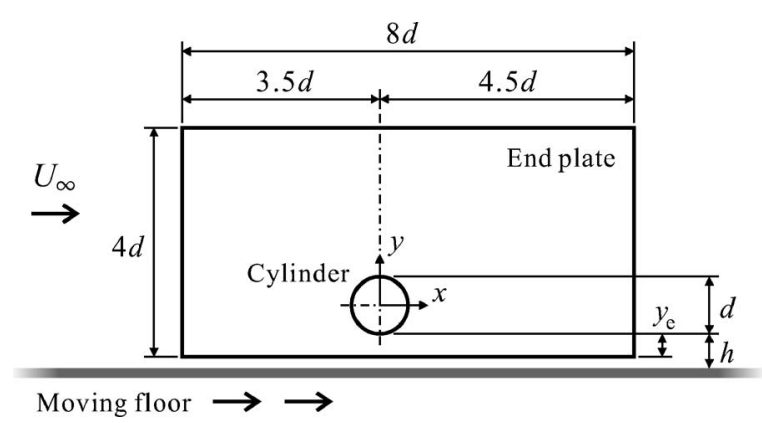

FIG. 1. Schematic of the cylinder model and end-plates.

\section{EXPERIMENTAL DETAILS}

\section{A. Wind tunnel, cylinder, and end-plates}

The time-averaged drag and lift measurements, surface oil flow visualization, and PIV measurements were performed in the $2.1 \times 1.5 \mathrm{~m}$ wind tunnel at the University of Southampton. The tunnel is of conventional closed circuit design, and the working section of $2.1 \mathrm{~m}$ wide $\times 1.5 \mathrm{~m}$ high $\times 4.4 \mathrm{~m}$ long is equipped with a large moving belt rig $(1.5 \mathrm{~m}$ wide $\times 3.2 \mathrm{~m}$ long) and also with a three-component overhead balance to measure the time-averaged forces. The moving floor works in conjunction with a boundary layer suction system, which ensures uniform airflow in the near-floor region. The freestream turbulence level is less than $0.3 \%$. A further description of the wind tunnel facility is given in Ref. 15 .

Figure 1 describes a standard layout of the circular cylinder model and the end-plates used in this study. The cylinder was placed above the moving floor with the axis lying parallel to the floor and perpendicular to the freestream. The cylinder model used is $6 \mathrm{~cm}$ in diameter and $50 \mathrm{~cm}$ in length: the aspect ratio $l / d=8.33$. The model is made of aluminum alloy and the surface is smooth; the relative roughness $K / d$, where $K$ is the estimated height of excrescences, is less than $0.01 \%$. Aluminum end-plates $3 \mathrm{~mm}$ thick (or Perspex end-plates $6.5 \mathrm{~mm}$ thick in the case of PIV measurements) were attached to both ends of the cylinder for the majority of the experiments. The size of the end-plates, given in Fig. 1, basically follows recommendations by Stansby, ${ }^{16}$ who optimized the distance from the leading edge of the plates to a cylinder placed in a freestream to provide an essentially two-dimensional flow around the cylinder. An additional factor to be considered here, however, is the gap between the end-plates and the moving floor since the plates are not allowed to contact the moving floor. In this study the distance from the bottom edge of the plates to the cylinder, denoted by $y_{e}$ in Fig. 1, was set at three different levels of 0 , $0.2 d$, and $0.4 d$ to examine the influence of the end-plate position, i.e., four different end conditions in total (three with end-plates and one without end-plates) were investigated in this study.

Figure 2 shows a photograph of the cylinder model and end-plates installed in the wind tunnel. The model was mounted on two steel struts, which were connected to the overhead balance of the wind tunnel. Two thin steel wires crossed between the struts to prevent the vibration of the

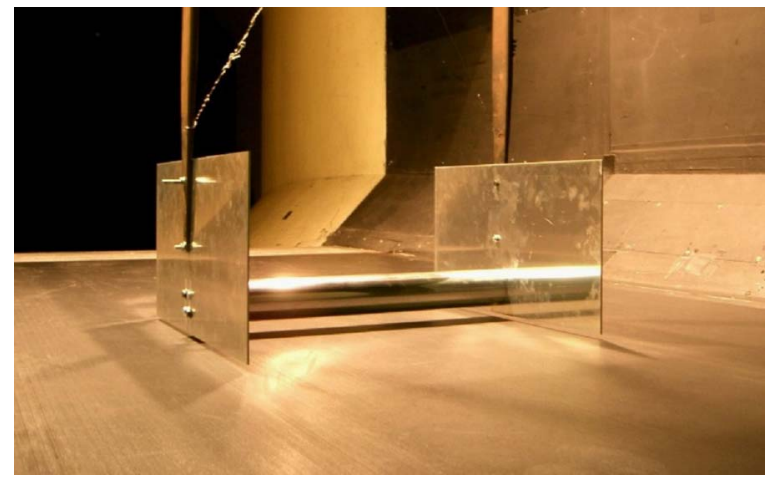

FIG. 2. Model installation in the wind tunnel.

model. The total blockage of the test section caused by the cylinder model, end-plates and struts was less than $3 \%$, and hence no corrections for blockage effects were made for the experimental results.

\section{B. Experimental procedure}

The time-averaged drag and lift measurements were performed as follows. First, the forces acting on the "total" package, consisting of the cylinder model, end-plates, struts and wires, were measured for each experimental condition (Reynolds numbers of 0.4 and $1.0 \times 10^{5}$, gap ratio $h / d$ from 0.05 to 2.0 , and the four end conditions). Second, the forces acting on the "tare" package, consisting only of the endplates, struts and wires, were measured for each condition. The drag and lift coefficients of the cylinder, $C_{D}$ and $C_{L}$, were then calculated by taking the difference between the two sets of measurements. The definitions of $C_{D}$ and $C_{L}$ are

$$
\begin{aligned}
& C_{D}=D / q_{\infty} l d, \\
& C_{L}=L / q_{\infty} l d,
\end{aligned}
$$

where $D$ and $L$ are the time-averaged drag and lift forces, respectively, and $q_{\infty}$ is the freestream dynamic pressure.

For the surface oil flow visualization, the cylinder model was painted matt black to obtain clear images of the oil flow pattern. A mixture of liquid paraffin and fine powder "Invisible Blue T70" was applied to the model surface, and then the tunnel was run for about $30 \mathrm{~min}$ to evaporate the paraffin, making the surface flow pattern visible and to be photographed. The oil flow tests were carried out for several $h / d$ and cylinder-end conditions, but only at the higher Reynolds number of $1.0 \times 10^{5}$.

The PIV measurements were performed using a Dantec FlowMap 2D-PIV system (PIV2100). A double-pulse $\mathrm{Nd}$ : YAG laser $(120 \mathrm{~mJ} /$ pulse $)$ was located approximately $1.5 \mathrm{~m}(25 d)$ downstream of the center of the cylinder to create a laser sheet of about $1 \mathrm{~mm}$ thick, illuminating the midspan, $x-y$ plane behind the cylinder. Smoke particles of about $1 \mu \mathrm{m}$ in size were used as tracer particles to be illuminated. The illuminated particle images were captured using a Dantec HiSense CCD camera $(1280 \times 1024$ pixels, 8 bits/pixel), which was synchronized with the laser so as to implement the so-called "double-frame/single-exposure" recording. ${ }^{17}$ The time delay between the two laser pulses was set at 


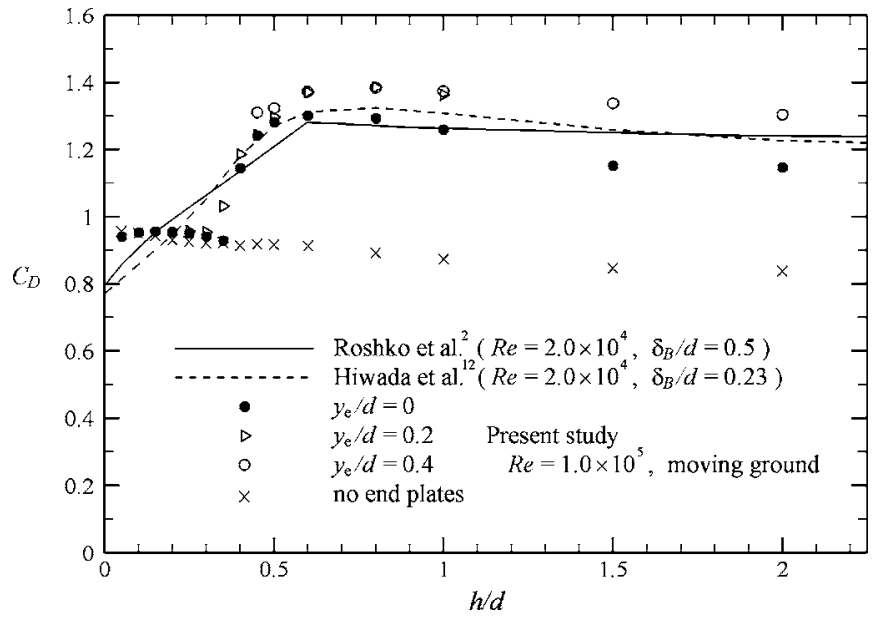

FIG. 3. Mean drag coefficient of a circular cylinder in the ground effect.

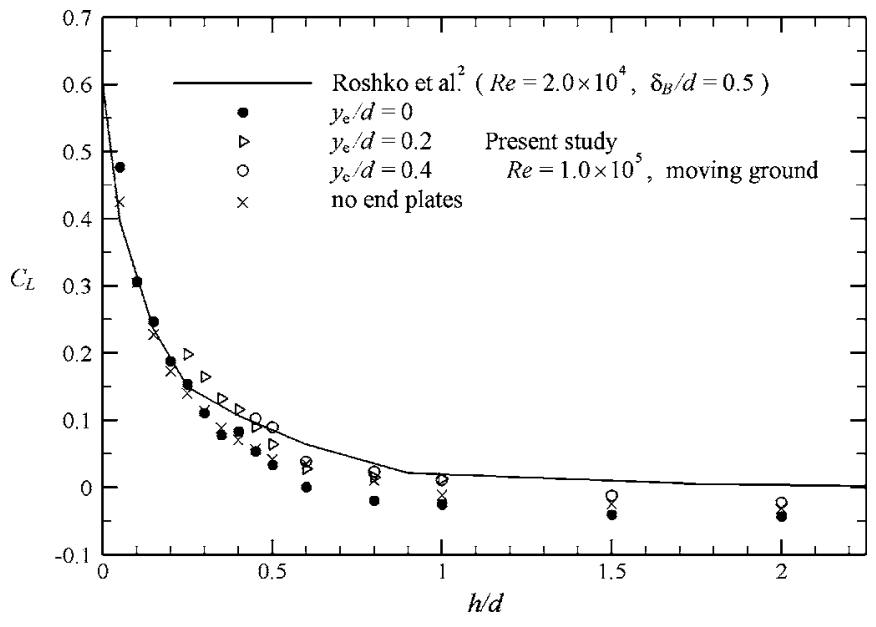

FIG. 4. Mean lift coefficient of a circular cylinder in the ground effect.
$50 \mu \mathrm{s}$, and 400 pairs of images were continuously recorded for each experimental condition with a sampling rate of $2 \mathrm{~Hz}$. Each pair of images was analyzed using a crosscorrelation technique with an interrogation area of $32 \times 32$ pixels with $50 \%$ overlapping in both horizontal and vertical directions. The resulting vectors were validated by the correlation-peak-height, velocity-range, and moving-average validations; ${ }^{18}$ the rejected vectors were replaced with interpolated values from the surrounding valid vectors. The PIV measurements were carried out only at $\mathrm{Re}=0.4 \times 10^{5}$.

\section{Uncertainties in measurements}

The random uncertainties or precision errors were estimated for the measurements of the time-averaged drag and lift coefficients, $C_{D}$ and $C_{L}$, following the theory of uncertainty analysis by Moffat. ${ }^{19}$ At the worst case under the condition of $\operatorname{Re}=0.4 \times 10^{5}$, the uncertainties in $C_{D}$ and $C_{L}$ were estimated to be \pm 0.016 and \pm 0.011 , respectively, with $95 \%$ confidence. Those for $\operatorname{Re}=1.0 \times 10^{5}$ were \pm 0.008 and \pm 0.003 , respectively. For the gap ratio $h / d$, an accuracy of \pm 0.002 was kept for the smallest $h / d$ of 0.05 , and \pm 0.01 was ensured for the other cases. A further description of the uncertainties is given in Ref. 20.

\section{EXPERIMENTAL RESULTS}

\section{A. Drag and lift coefficients}

The variations of the time-averaged drag coefficient of the cylinder in the ground effect are shown in Fig. 3 for the four different cylinder-end conditions investigated. The results of some of the earlier studies reviewed in Sec. I are also included in this figure for comparison. For the present results, only those for $\mathrm{Re}=1.0 \times 10^{5}$ are shown here since there were no substantial differences in results between the two Reynolds numbers tested. As can be seen from the figure, for all three cases where the end-plates were attached to the cylinder $\left(y_{e} / d=0,0.2\right.$, and 0.4$)$, a rapid reduction in $C_{D}$ occurred as $h / d$ decreased to less than about 0.5 , similar to the results by Roshko et $a l^{2}$ and Hiwada et al., ${ }^{12}$ who used a fixed ground rather than a moving ground. The drag reduc- tion observed in the present study, however, suddenly stopped around $h / d=0.35$ and then $C_{D}$ remained almost constant at slightly less than 1 as the cylinder came close to the ground. As will be shown later, the critical change in $C_{D}$ around $h / d=0.35$ appears to coincide with the global cessation of the large-scale Kármán-type vortex shedding behind the cylinder.

As concerns the influence of the position of the endplates, there is a certain difference between the results for $y_{e} / d=0$ and 0.2 especially at larger gap ratios of $h / d>0.5$, but little difference between $y_{\mathrm{e}} / d=0.2$ and 0.4 . This is presumably because the effectiveness of the end-plates is reduced as the distance from the bottom edge of the plates to the cylinder, $y_{\mathrm{e}}$, is decreased close to zero. At the largest gap ratio of $h / d=2$, the drag coefficient of the cylinder with endplates at $y_{\mathrm{e}} / d=0.4$ was found to be 1.3 , which is comparable to that for a long circular cylinder in a freestream (i.e., outside the ground effect) in the upper-subcritical flow regime ${ }^{3}$ and therefore suggests the full effectiveness of the end-plates in this case.

It can also be seen from Fig. 3 that the drag behavior of the cylinder without end-plates is totally different from that for the cylinder with end-plates: the drag gradually decreased, with no critical change, as the gap ratio increased. At $h / d=2$, the drag coefficient for the cylinder without endplates was found to be 0.85 , which is consistent with that for a short circular cylinder of similar aspect ratio in a freestream. ${ }^{21}$ Of interest is that there is no substantial effect of the end conditions of the cylinder at smaller gap ratios $(h / d<0.35)$, where $C_{D}$ showed an almost constant value of 0.95 for the cylinder with end-plates at $y_{e} / d=0$ and 0.2 , and also for the cylinder without end-plates.

Figure 4 shows the variations of the mean lift coefficient of the cylinder in the ground effect at $\mathrm{Re}=1.0 \times 10^{5}$ for the four different end conditions. Similar but a little more scattered variations of $C_{L}$ were observed at the lower Reynolds number of $0.4 \times 10^{5}$, details of which are not provided here. Instead, the results for a fixed ground by Roshko et al. ${ }^{2}$ are shown in the same figure for comparison. It can be seen that both the ground conditions (moving or fixed) and the end conditions (with or without end-plates) have only minor ef- 
With end plates $\left(y_{\mathrm{e}} / d=0\right)$

$h / d=1.0$

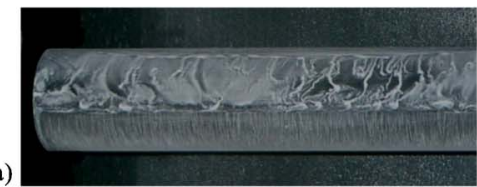

(a)

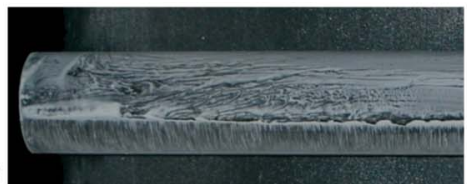

(c)

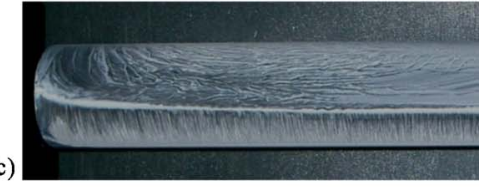

(d)

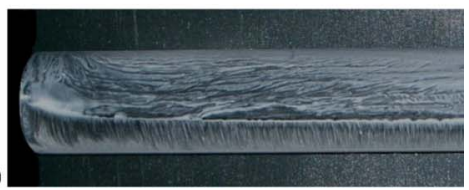

FIG. 5. Oil flow patterns on the upper surface of the cylinder with and without end-plates, $\operatorname{Re}=1.0 \times 10^{5}$. fects on the lift behavior of the cylinder; $C_{L}$ rapidly increases as $h / d$ decreases to less than about 0.5 . This little sensitivity of $C_{L}$ to the ground conditions agrees with the observation by Hiwada et al. $^{12}$ that the thickness of the boundary layer formed on the fixed ground had little influence on the lift behavior of the cylinder.

\section{B. Surface oil flow pattern}

Figures 5 and 6 show, respectively, the oil flow patterns on the upper (open-side) and bottom (gap-side) surfaces of the cylinder, with and without end-plates, observed at two different gap ratios. Note that only half of the cylinder span is shown here since the oil flow patterns appeared to be symmetric in the spanwise direction for all cases tested. The airflow direction is from downside to upside in Fig. 5, and is from upside to downside in Fig. 6.

As is obvious from the orderly oil flow patterns in the upstream region, for all cases, the airflow remains laminar and almost two dimensional before the separation, i.e., the flow is subcritical. The separation line on the upper surface of the cylinder is straight when with end-plates at $y_{e} / d=0$ [Figs. 5(a) and 5(b)], and is slightly curved near the ends when without end-plates [Figs. 5(c) and 5(d)]; a similar pattern of this slightly curved separation near the free ends has been reported for a circular cylinder of finite length in a freestream (outside the ground effect). ${ }^{21}$ On the bottom surface of the cylinder in the ground effect, however, the separation line remains straight even without end-plates [Figs. 6(c) and 6(d)].

It can also be seen from the oil flow patterns that the position of the flow separation moves upstream and downstream on the upper and bottom surfaces, respectively, as the cylinder comes close to the ground. Figure 7 summarizes the separation angle $\theta_{\text {sep }}$ [the angle from the front $(x / d=-0.5$, $y / d=0)$ to the separation point estimated from the oil flow patterns at the midspan of the cylinder] for all gap ratios investigated. As has been suggested by Bearman and Zdravkovich $^{4}$ for a circular cylinder near a fixed ground, the lift variation of the cylinder in ground effect (cf. Fig. 4) can be largely explained by this angular shift of the flow field around the cylinder.

\section{Near wake structure \\ 1. Cylinder with end-plates}

Figure 8 shows the time-averaged, midspan $(z / d=0)$ flow field data, obtained from 400 samples of PIV data for each case, behind the cylinder with end-plates at $y_{e} / d=0$. The time-averaged velocity vectors, streamwise velocity contours, and spanwise vorticity contours are depicted in Figs. 8(a)-8(1), respectively, for four different $h / d$ of 0.6 , $0.4,0.3$, and 0.2 . Note that the velocity is nondimensionalized by the freestream velocity $U_{\infty}$, and the vorticity is also nondimensionalized by $U_{\infty}$ and the cylinder diameter $d$ as

$$
\omega_{z}=(\partial V / \partial x-\partial U / \partial y) d / U_{\infty}
$$

Also note that the data close to the cylinder and ground surfaces have been discarded as they were disturbed by the reflection of light from the surfaces. It can be seen from the figures that the recirculation region behind the cylinder is significantly elongated as the gap ratio $h / d$ is reduced from 0.6 to 0.3 , but no substantial difference can be seen between $h / d=0.3$ and 0.2 . It is also observed that the two separated shear layers are deflected slightly upward as the cylinder comes close to the ground.
With end plates $\left(y_{\mathrm{e}} / d=0\right)$

$h / d=1.0$

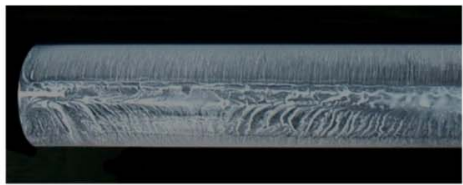

(a)

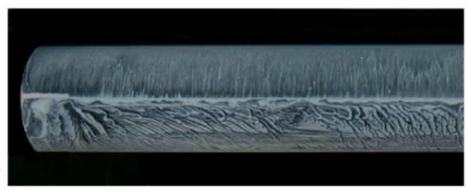

Without end plates

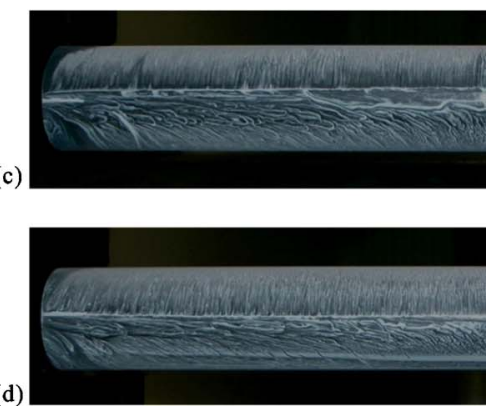

FIG. 6. Oil flow patterns on the bottom surface of the cylinder with and without end-plates, $\operatorname{Re}=1.0 \times 10^{5}$. 


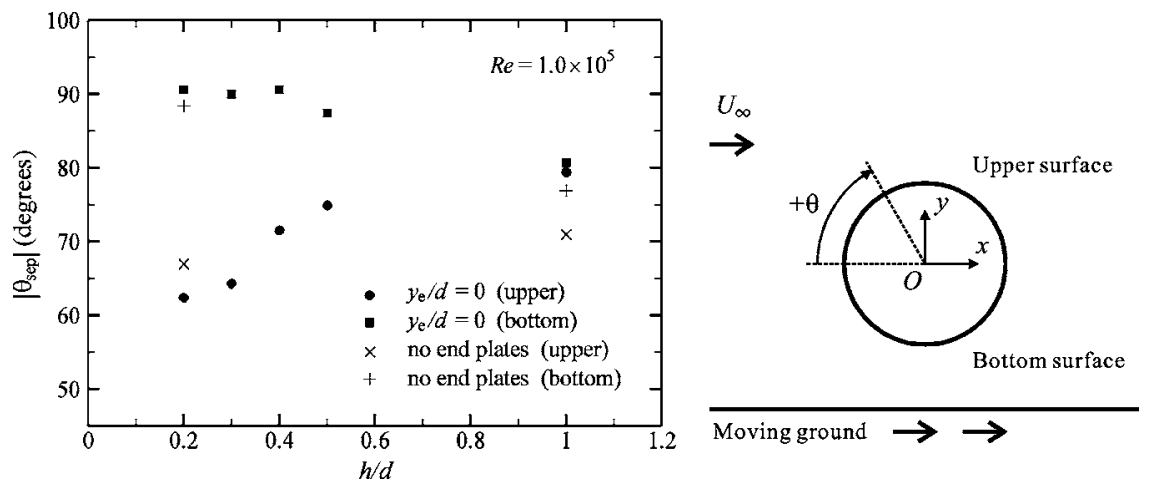

FIG. 7. Separation angle vs gap ratio for the cylinder with and without end-plates.
More details of the near wake structure can be seen from the instantaneous flow field data behind the cylinder. Figures 9(a) and 9(b) show typical instantaneous spanwise vorticity contours at $h / d=0.6$ and 0.2 , respectively. At $h / d=0.6$, large-scale Kármán-type (alternating) vortices are generated just behind the cylinder, which is a common feature of the wake of a circular cylinder in a freestream (i.e., outside the ground effect) in the upper-subcritical flow regime. ${ }^{3}$ At $h / d$ $=0.2$, however, such large-scale vortices are not generated and instead the so-called "dead-fluid" zone is created behind the cylinder, bounded by two nearly parallel shear layers each producing only small-scale vortices in the near wake region. Of further interest is that, at an "intermediate" gap ratio of 0.4 , the Kármán-type vortex shedding was found to be intermittent in the near wake region. That is, both types of instantaneous wake structures of Figs. 9(a) and 9(b) were frequently observed throughout the measurements at this gap ratio. Although the time evolution of the wake structure was not able to be captured in this study due to the low sampling rate of the PIV recording, a statistical observation on this intermittency can be made by plotting the positions of vortex cores identified as follows.

Figure 10 shows the contours of the (two-dimensional) swirl strength $\lambda_{c i}$, that is the imaginary part of the complex eigenvalue of the local velocity gradient tensor, ${ }^{22}$ for the two instantaneous flow fields at $h / d=0.6$ and 0.2 discussed above. Note that the local velocity gradient tensor $\underline{\underline{D}}^{2 \mathrm{D}}$ is defined as

$$
\underline{\underline{D}}^{2 \mathrm{D}}=\left[\begin{array}{ll}
\frac{\partial U}{\partial x} & \frac{\partial U}{\partial y} \\
\frac{\partial V}{\partial x} & \frac{\partial V}{\partial y}
\end{array}\right] .
$$

Also note that the symbols $(\diamond)$ in the figures indicate the positions of vortex cores, which were automatically identified by the positions of the peaks of the swirl strength that were above an arbitrary threshold of $0.8 \mathrm{~s}^{-1}$. Again, the difference in the near wake structure between the two cases can be seen from the figure; the large-scale Kármán-type vortices due to the wake instability are generated at $h / d=0.6$, whereas only small-scale vortices due to the shear layer instability are formed at $h / d=0.2$.

Figure 11 shows the plots of the vortex cores, which were extracted from 100 instantaneous PIV data and then superimposed onto one figure for each case, for four different gap ratios of $0.6,0.4,0.3$, and 0.2 . These plots clearly show that the Kármán-type vortex shedding is totally suppressed and a dead-fluid zone is formed behind the cylinder at the smaller $h / d$ of 0.2 and 0.3 , and also show that the number of the vortex cores captured behind the cylinder at $h / d=0.4$ is less than that at $h / d=0.6$, i.e., the Kármán-type vortex shedding is intermittent at $h / d=0.4$.

\section{Cylinder without end-plates}

Figure 12 shows the time-averaged, midspan $(z / d=0)$ flow field data for the cylinder without end-plates. The timeaveraged velocity vectors, streamwise velocity contours, and spanwise vorticity contours are described in Figs. 12(a)-12(f), respectively, for two different $h / d$ of 0.6 and 0.2 . Then the plots of the vortex cores for the two gap ratios, again extracted from 100 instantaneous PIV data for each case, are given in Fig. 13. Note that here the data in the upper region of the cylinder as well as in the vicinity of the cylinder and ground surfaces have been discarded as they were disturbed by the reflection of light. In contrast to the results for the cylinder with end-plates, there is little difference in the near wake structure of the cylinder between the two gap ratios when without end-plates; the large-scale Kármán-type vortices are not generated and a large recirculation region is formed behind the cylinder, even at the larger gap ratio of 0.6. Although the PIV measurements were carried out only at the midspan of the cylinder in this study, it is inferred that the Kármán-type vortex shedding behind the cylinder without end-plates was suppressed not only due to the influence of the nearby ground but also due to the influence of the tip vortices generated from the two free ends of the cylinder. ${ }^{21}$ Further investigations are needed, however, to fully understand this mechanism since only one aspect ratio of the cylinder $(l / d=8.33)$ was investigated in this study.

Figure 14 shows a comparison of the time-averaged streamwise velocity profiles between the cylinders with and without end-plates, for (a) $h / d=0.6$, and (b) $h / d=0.2$. Note that the profiles at $x / d=1.0,1.5,2.0$, and 2.5 on the midspan plane $(z / d=0)$ are plotted in these figures. The effects of the end-plates on the midspan velocity profiles are significant at $h / d=0.6$ because the Kármán-type vortex shedding occurs if the plates are used at this gap ratio, whereas no substantial effect can be seen at $h / d=0.2$ as the shedding does not occur for both cases at this gap ratio. 


$$
\rightarrow:|\boldsymbol{U}| / U_{\infty}=1
$$
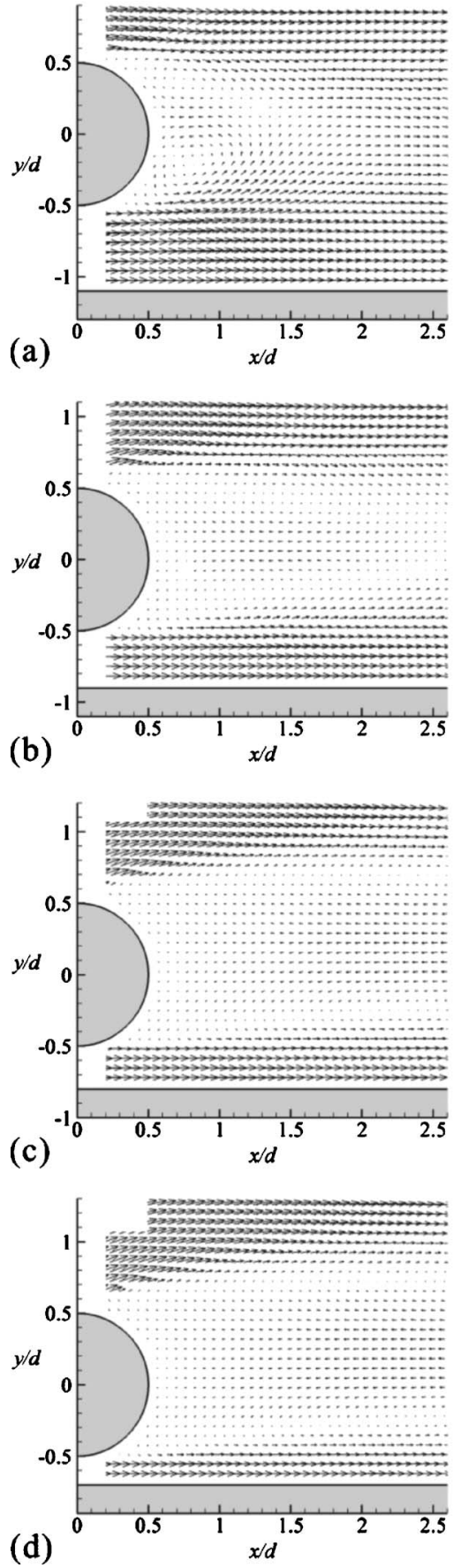

$U / U_{\infty}$
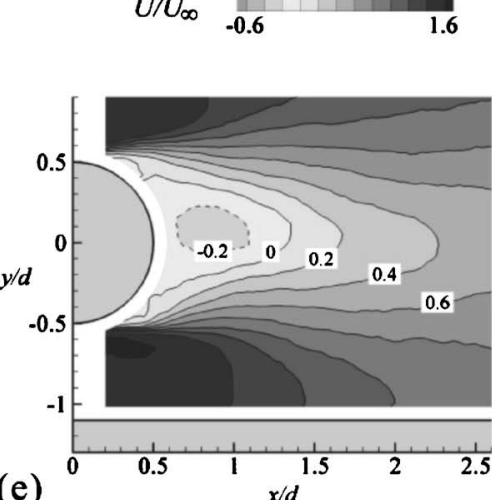

(e)

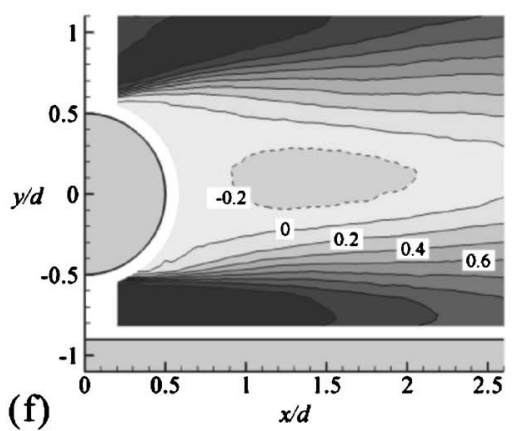

(f)

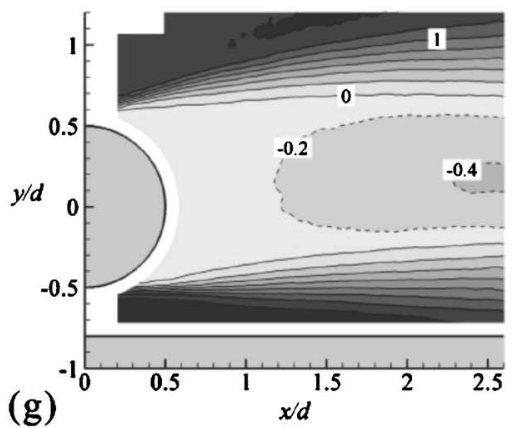

(g)

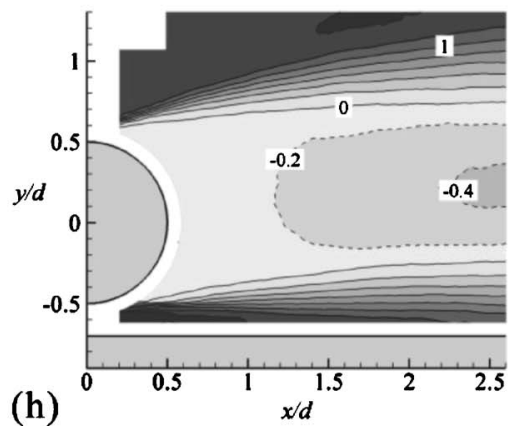

$\omega_{z} \quad-12$
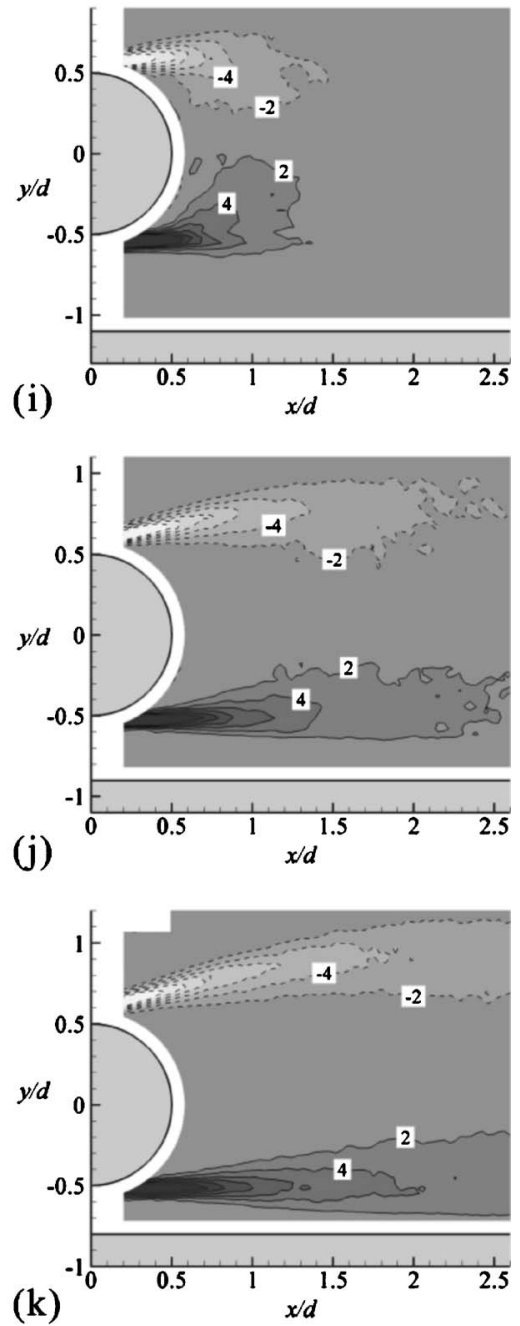

(k)

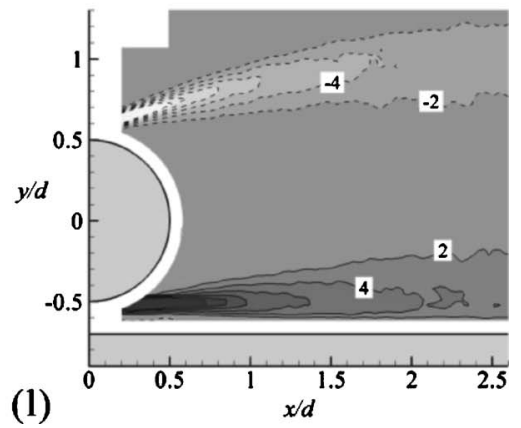

FIG. 8. Time-averaged flow fields behind the cylinder with end-plates $\left(y_{e} / d=0\right)$ : $(\mathrm{a}-\mathrm{d})$ velocity vectors, $(\mathrm{e}-\mathrm{h})$ contours of streamwise velocity, and (i-1) contours of spanwise vorticity, at four different $h / d$ of $(\mathrm{a}, \mathrm{e}, \mathrm{i}) 0.6$, (b, f, j) $0.4,(\mathrm{c}, \mathrm{g}, \mathrm{k}) 0.3$, and $(\mathrm{d}, \mathrm{h}, \mathrm{l}) 0.2 ; \operatorname{Re}=0.4 \times 10^{5}$.

\section{DISCUSSION OF RESULTS}

In the following, further discussions will be made on: (i) the relation between the cessation of the Kármán vortex shedding and the drag reduction of a circular cylinder in the ground effect, (ii) the cause of the cessation of the shedding in the ground effect, and (iii) the intermittency of the shedding in the ground effect. Note that the discussions here are based on the results obtained for the cylinder with endplates.
As concerns the first point, it can be concluded from the results that the drag reduction of a circular cylinder in the ground effect is directly related to the cessation of the Kármán-type vortex shedding, as far as the thickness of the boundary layer on the ground is negligible or sufficiently small (note that in fact a very thin boundary layer still locally existed on the moving ground in this study since the ground was running at the same speed as the freestream rather than as the local flow through the gap between the cylinder and 

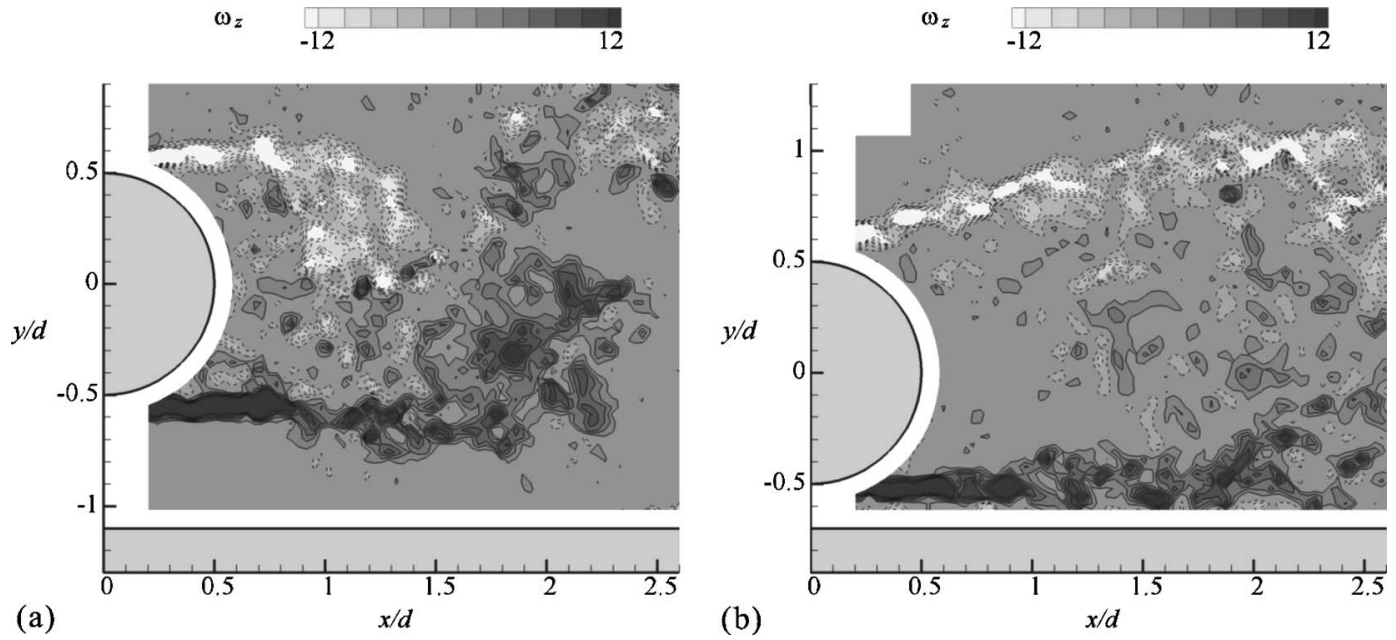

FIG. 9. Typical instantaneous spanwise vorticity contours behind the cylinder with end-plates $\left(y_{e} / d=0\right)$ : (a) $h / d=0.6$, and (b) $h / d=0.2 ; \operatorname{Re}=0.4 \times 10^{5}$.

the ground). It can be confirmed from Figs. 3 and 11 that, for the cylinder with end-plates, the gap regime in which the Kármán vortex shedding is continuously observed [cf. Fig. $11(\mathrm{a})]$ corresponds to the regime of higher $C_{D}(h / d>0.5$, cf. Fig. 3), whereas the regime in which the shedding is totally suppressed [cf. Figs. 11(c) and 11(d)] corresponds to the regime of lower $C_{D}(h / d<0.35$, cf. Fig. 3). This direct relationship might be relatively straightforward to understand since in general the Kármán-type vortices behind a bluff body continuously draw in fluid from the base region of the body during their growth, and this entrainment process sustains a low base pressure and therefore a high drag force acting on the body, for example as demonstrated by Bearman and Trueman. ${ }^{23}$ Although the base pressure of the cylinder was not measured, the critical drag reduction of the cylinder observed in the present study could also be reasonably explained by this mechanism. It is also consistent with this explanation that the reduction of the time-averaged $C_{D}$ occurs in the intermediate gap regime $(0.35<h / d<0.5$, cf. Fig. 3) and it is in this regime that the Kármán vortex shedding was found to become intermittent.
Another important conclusion confirmed by using a moving ground in this study should be the fact itself that the Kármán-type vortex shedding from the cylinder did cease at $h / d<0.3$ to 0.4 despite a sufficient amount of flow through the gap between the cylinder and the moving ground [note that the flow through the gap is not choked but accelerated at $h / d=0.3$ or even 0.2 , cf. Figs. $8(\mathrm{~g})$ and $8(\mathrm{~h})$, for example], and this leads to the second point of the discussion, that is, the cause of the cessation of the Kármán vortex shedding in ground effect. It might still be possible to argue that the very thin boundary layer locally developed on the moving ground generates vorticity of opposite sign and thus to interfere with the separated shear layer from the bottom side of the cylinder, just like the cases with a thick boundary layer formed on a fixed ground. ${ }^{8,24}$ Judging from the mean spanwise vorticity contours [cf. Figs. 8(k) and 8(l)], however, the vorticity of the shear layer from the bottom side of the cylinder appears to be as strong as that from the upper side of the cylinder; there should be another explanation to the cessation of the shedding in ground effect.

In general (i.e., for general two-dimensional bluff bodies
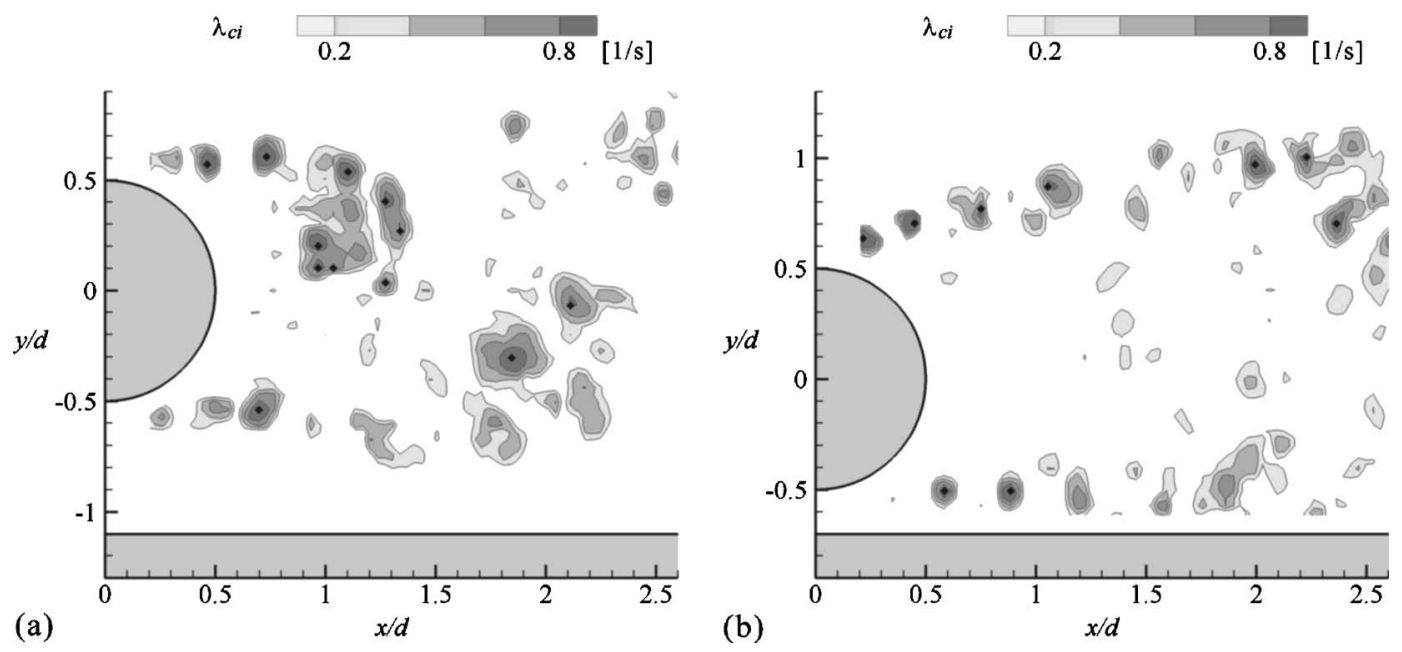

FIG. 10. Instantaneous swirl strength $\lambda_{c i}$ contours behind the cylinder with end-plates $\left(y_{e} / d=0\right)$ : (a) $h / d=0.6$, and (b) $h / d=0.2 ; \operatorname{Re}=0.4 \times 10^{5}$. Symbols ( indicate the positions of vortex cores identified by the peaks of swirl strength with $\lambda_{c i}>0.8 \mathrm{~s}^{-1}$. 


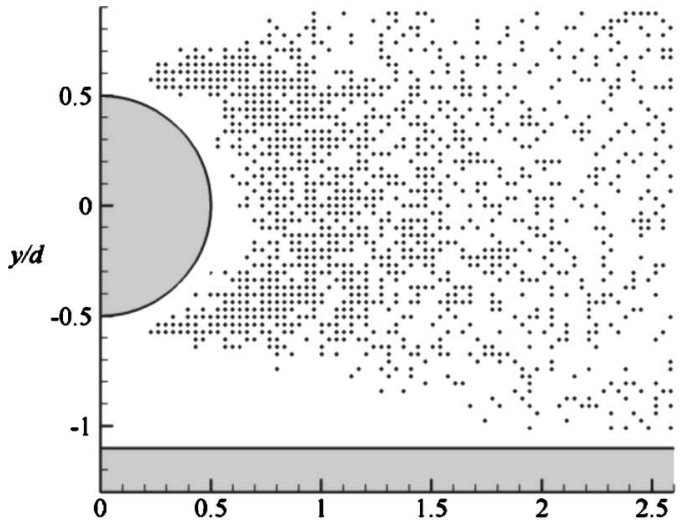

(a)

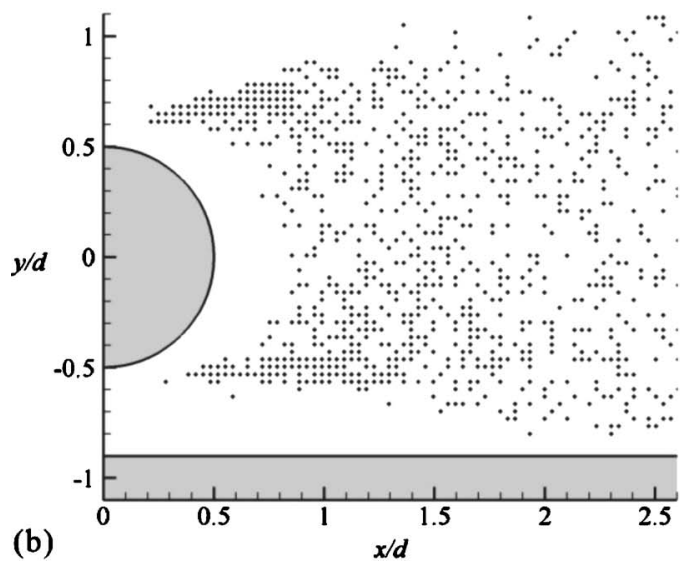

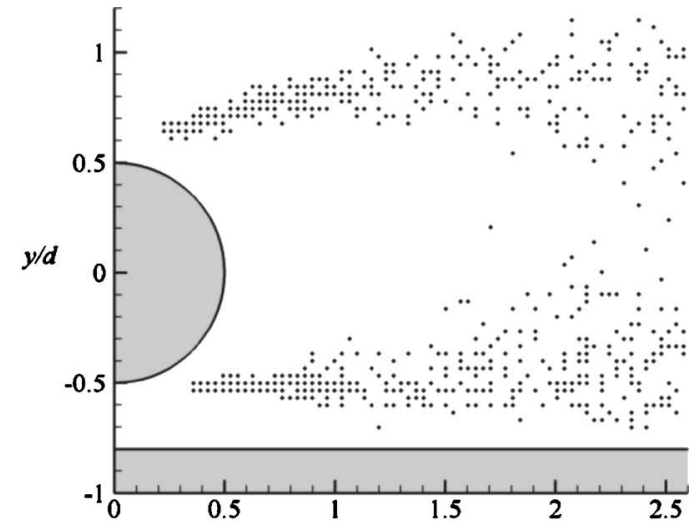

(c)

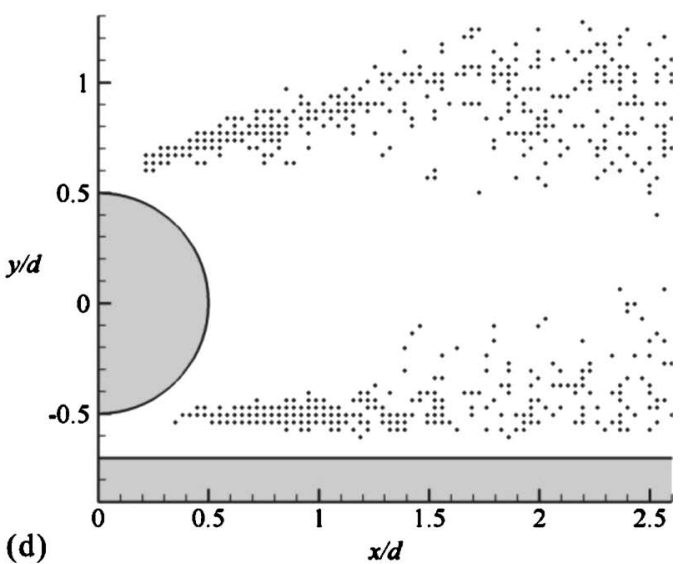

FIG. 11. Positions of vortex cores (extracted from 100 instantaneous PIV data for each case) behind the cylinder with end-plates $\left(y_{e} / d=0\right)$ : (a) $h / d=0.6,(b)$ $h / d=0.4$, (c) $h / d=0.3$, and (d) $h / d=0.2 ; \operatorname{Re}=0.4 \times 10^{5}$.

$$
\rightarrow:|\boldsymbol{U}| / U_{\infty}=1
$$
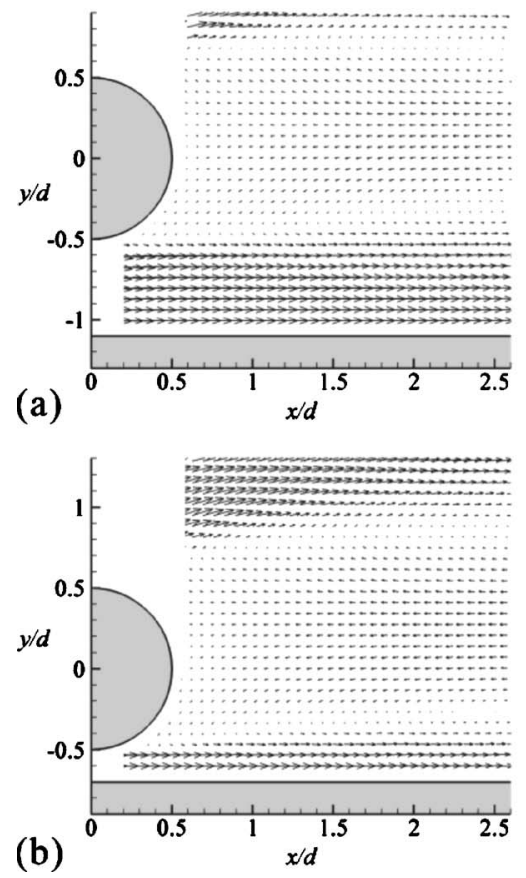

$U / U_{\infty}$
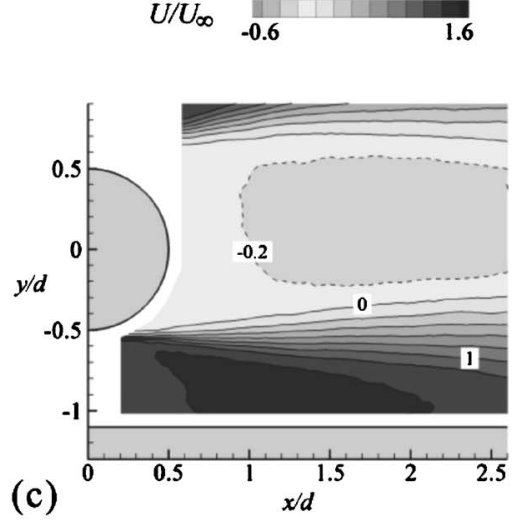

(c)

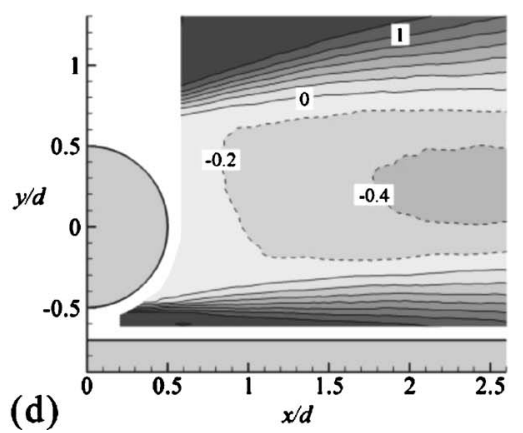

$\omega=-12$
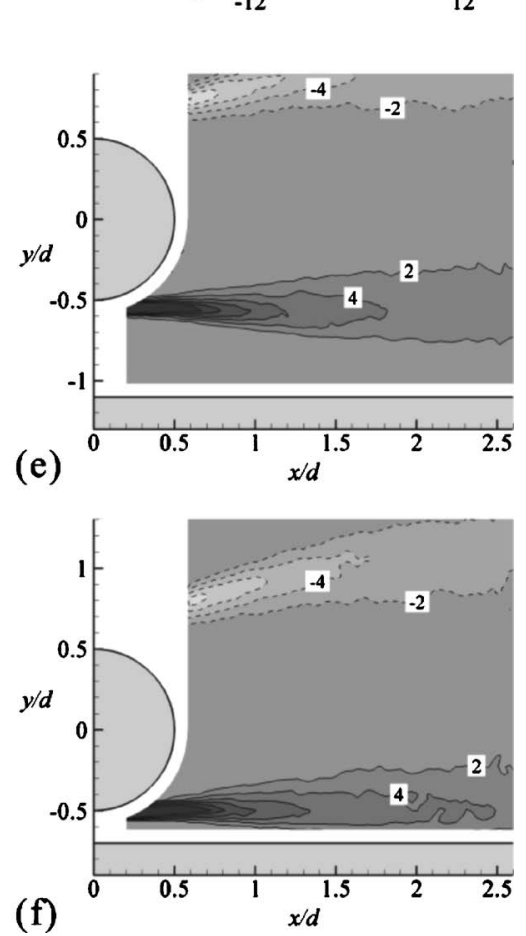

(f)

FIG. 12. Time-averaged flow fields behind the cylinder without end-plates: (a, b) velocity vectors, (c, d) contours of streamwise velocity, and (e, f) contours of spanwise vorticity, at two different $h / d$ of (a, c, e) 0.6 , and (b, d, f) $0.2 ; \operatorname{Re}=0.4 \times 10^{5}$. 

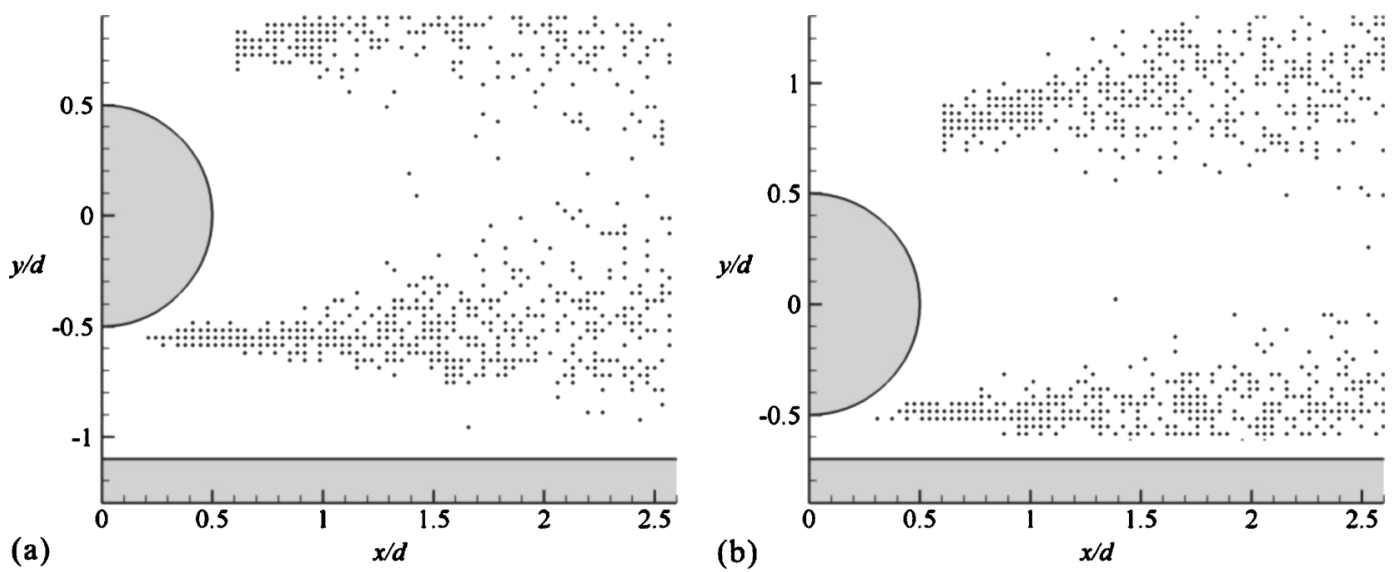

FIG. 13. Positions of vortex cores (extracted from 100 instantaneous PIV data for each case) behind the cylinder without end-plates: (a) $h / d=0.6$, and (b) $h / d=0.2 ; \operatorname{Re}=0.4 \times 10^{5}$.

in a uniform cross-flow), the formation of the Kármán vortices is due to the wake instability caused by the communication between the two shear layers, and this can be confirmed, for example, by the cessation of the shedding behind a cylinder equipped with a backward splitter plate of sufficient length, which was first experimentally observed by Roshko. $^{25}$ Recent studies show that this wake instability could be explained by the existence of an absolutely unstable region in the near wake region, which allows local disturbances to propagate both upstream and downstream and thus to produce a resonance between the traveling instability waves (see Refs. 26 and 27 for comprehensive reviews). From this point of view, it can be explained that the splitter plate mentioned above prevents the resonance and hence the resulting Kármán vortex shedding as the plate changes the state of the near wake from being absolutely unstable to being convectively unstable. Of interest here is that a similar explanation might also be applicable to the cessation of the shedding in ground effect observed in this study, i.e., the existence of the ground somehow restricts the propagation of disturbances in the near wake region and thus to prevent the resulting Kármán vortex shedding. A linear stability analysis currently being conducted by the present authors indicates that the near wake of the cylinder is changed from being absolutely unstable to being convectively unstable as the cyl- inder comes close to the ground; further investigations are required, however, to fully resolve this issue.

The third and final point of the discussion here is the intermittency of the Kármán vortex shedding in the intermediate gap regime, which may also be of some relevance to the mechanisms of the cessation of the shedding discussed above. A similar intermittency of the shedding in an intermediate gap regime has been observed by Martinuzzi et al. ${ }^{28}$ for a square cylinder placed near a fixed ground. They suggested, however, that the intermittency of the shedding behind the square cylinder is due to the intermittency of the reattachment of the separated shear layer (from the bottom leading edge) on the bottom surface, which is not the case for a circular cylinder in the ground effect. Bearman and Zdravkovich ${ }^{4}$ also suggested the intermittency of the shedding for a circular cylinder placed near a fixed ground, but the mechanism of this intermittency is still unexplained. A possible clue to the mechanism may be found in the recent experiments by Zhang et al. ${ }^{29}$ for a half cylinder placed near a moving ground (with the flat surface of the cylinder facing downstream), where the shedding was suddenly suppressed and the drag coefficient of the cylinder was suddenly dropped from about 1.5 to 1 as the gap ratio $h / d$ was reduced from 0.55 to 0.525 . They reported that the flow separation from the bottom side of the half cylinder was fixed at the
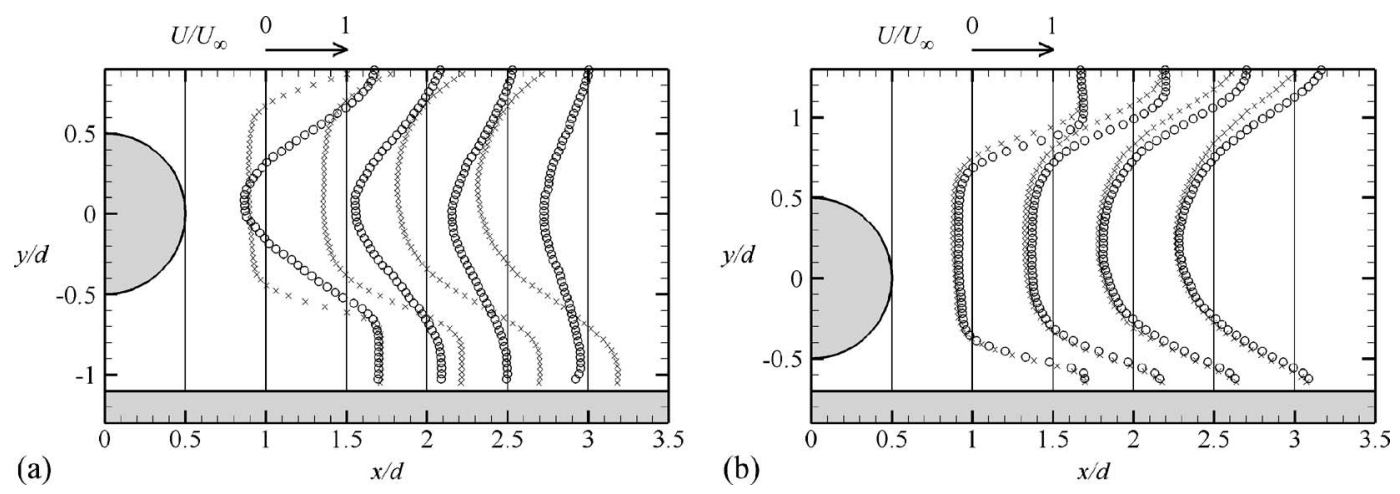

FIG. 14. Mean streamwise velocity profiles behind the cylinder: (a) $h / d=0.6$, and (b) $h / d=0.2 ; \operatorname{Re}=0.4 \times 10^{5}$. Circles $(O)$ show the results for the cylinder with end-plates $\left(y_{e} / d=0\right)$, and cross marks $(\times)$ show the results for the cylinder without end-plates. 
cylinder edge (i.e., the separation angle $\left|\theta_{\text {sep }}\right|=90^{\circ}$ ) when $h / d \leq 0.525$, whereas that measured on the circular cylinder in the present study at $h / d=0.4$ (where the intermittency of the shedding occurs) is slightly greater than $90^{\circ}$ (cf. Fig. 7) and presumably is not fixed but fluctuates in time due to the smooth geometry. Considering the other similarities between the two experiments, it is inferred that the fluctuation or unsteadiness of the flow separation point should be of some importance to the intermittency of the shedding from the circular cylinder in the intermediate gap regime.

\section{CONCLUSIONS}

The characteristics of the flow around a circular cylinder placed near and parallel to a moving ground were experimentally investigated at two upper-subcritical Reynolds numbers of 0.4 and $1.0 \times 10^{5}$. The experiments were performed using a moving ground running at the same speed as the freestream in order to avoid the confusing effects of the boundary layer formed on the ground and thereby to reveal the essence of the ground effect. The influence of the spanwise-end condition of the cylinder (the aspect ratio $/ / d$ $=8.33$ in this study) was also examined by conducting the experiments with and without end-plates. The main conclusions obtained are as follows.

For the cylinder with end-plates, on which the surface oil flow patterns were observed to be essentially twodimensional, significant effects of the gap ratio were observed on the near wake structure and also on the timeaveraged drag coefficient. The flow characteristics may be classified into three gap regimes: large-gap $(h / d>0.5)$, intermediate-gap $(0.35<h / d<0.5)$, and small-gap $(h / d$ $<0.35$ ) regimes. In the large-gap regime, large-scale Kármán-type vortices were generated just behind the cylinder, resulting in higher drag coefficients of about 1.3 as the vortices continuously entrained air from the base region of the cylinder. In the intermediate-gap regime, the Kármán vortex shedding became intermittent, and hence the timeaveraged drag coefficient rapidly decreased as $h / d$ was reduced from 0.5 to 0.35 . In the small-gap regime, the shedding totally ceased and instead a dead-fluid zone was created, bounded by two nearly parallel shear layers each producing only small-scale vortices. Of particular interest here was that there was little influence of $h / d$ on the drag coefficient of the cylinder in the small-gap regime: $C_{D}$ was almost constant at a lower value of slightly less than 1 for $h / d<0.35$, unlike with that usually observed near a fixed ground. These results suggest that the drag reduction of a circular cylinder in ground effect is directly related to the cessation of the Kármán vortex shedding if the thickness of the boundary layer on the ground is sufficiently small.

For the cylinder without end-plates, on the other hand, no such significant effects of $h / d$ were observed either on the near wake structure or on the drag coefficient. The Kármántype vortices were not generated in the near wake region, resulting in a nearly constant, lower drag coefficient of 0.85 to 0.95 for a wide $h / d$ range from 2.0 to 0.05 . A dead-fluid zone was continuously formed and thus a large recirculation region was created behind the cylinder at both larger and smaller gap ratios of 0.6 and 0.2 .

Discussions were also given to the essential cause of the cessation of the Kármán vortex shedding in the ground effect. A very thin boundary layer still locally existed on the moving ground in this study and hence it might still be possible to argue that this thin boundary layer generates vorticity of opposite sign to interfere with the separated shear layer from the bottom side of the cylinder (just like the cases with a thick boundary layer formed on a fixed ground). A more probable explanation to the cessation of the shedding observed in this study, however, is that the ground restricts the propagation of disturbances and thus prevents a resonance between the traveling instability waves in the near wake region, similar to the mechanism for the cessation of the shedding behind a cylinder equipped with a backward splitter plate. Further investigations are required, however, to fully resolve this issue.

\section{ACKNOWLEDGMENTS}

T. Nishino is supported by a research scholarship from the School of Engineering Sciences, University of Southampton, United Kingdom. The authors would like to thank Mr. Mike Tudor-Pole and Mr. Martijn van den Berg for their assistance in performing the experiments.

${ }^{1}$ S. Taneda, "Experimental investigation of vortex streets," J. Phys. Soc. Jpn. 20, 1714 (1965).

${ }^{2}$ A. Roshko, A. Steinolfson, and V. Chattoorgoon, "Flow forces on a cylinder near a wall or near another cylinder," in Proceedings of the 2nd U.S. National Conference on Wind Engineering Research, Colorado State University, Fort Collins, 1975, paper IV-15.

${ }^{3}$ M. M. Zdravkovich, Flow Around Circular Cylinders: Vol 1: Fundamentals (Oxford University Press, Oxford, 1997), Chap. 1.

${ }^{4}$ P. W. Bearman and M. M. Zdravkovich, "Flow around a circular cylinder near a plane boundary," J. Fluid Mech. 89, 33 (1978).

${ }^{5}$ S. J. Price, D. Sumner, J. G. Smith, K. Leong, and M. P. Paidoussis, "Flow visualization around a circular cylinder near to a plane wall," J. Fluids Struct. 16, 175 (2002).

${ }^{6} \mathrm{G}$. Buresti and A. Lanciotti, "Vortex shedding from smooth and roughened cylinders in cross-flow near a plane surface," Aeronaut. Q. 30, 305 (1979).

${ }^{7}$ F. Angrilli, S. Bergamaschi, and V. Cossalter, "Investigation of wall induced modifications to vortex shedding from a circular cylinder," ASME J. Fluids Eng. 104, 518 (1982).

${ }^{8}$ A. J. Grass, P. W. J. Raven, R. J. Stuart, and J. A. Bray, "Influence of boundary layer velocity gradients and bed proximity on vortex shedding from free spanning pipelines," ASME J. Energy Resour. Technol. 106, 70 (1984).

${ }^{9}$ M. M. Zdravkovich, "Observation of vortex shedding behind a towed circular cylinder near a wall," in Flow Visualization III (Proceedings of the 3rd International Symposium on Flow Visualization), edited by W. J. Yang (Hemisphere, Washington, D.C., 1985), pp. 423-427.

${ }^{10}$ C. Lin, W.-J. Lin, and S.-S. Lin, "Flow characteristics around a circular cylinder near a plane boundary," in Proceedings of the 16th International Symposium on Transport Phenomena (ISTP-16), Prague, Czech Republic, 2005, CD-ROM.

${ }^{11}$ M. M. Zdravkovich, "Forces on a circular cylinder near a plane wall," Appl. Ocean. Res. 7, 197 (1985).

${ }^{12}$ M. Hiwada, I. Mabuchi, M. Kumada, and H. Iwakoshi, "Effect of the turbulent boundary layer thickness on the flow characteristics around a circular cylinder near a plane surface" (in Japanese), Trans. Jpn. Soc. Mech. Eng., Ser. B 52, 2566 (1986). 
${ }^{13}$ C. Lei, L. Cheng, and K. Kavanagh, "Re-examination of the effect of a plane boundary on force and vortex shedding of a circular cylinder," J. Wind. Eng. Ind. Aerodyn. 80, 263 (1999).

${ }^{14}$ M. M. Zdravkovich, Flow Around Circular Cylinders: Vol 2: Applications (Oxford University Press, Oxford, 2003), pp. 880-881.

${ }^{15}$ K. Burgin, P. C. Adey, and J. P. Beatham, "Wind tunnel tests on road vehicle models using a moving belt simulation of ground effect," J. Wind. Eng. Ind. Aerodyn. 22, 227 (1986).

${ }^{16}$ P. K. Stansby, "The effects of end plates on the base pressure coefficient of a circular cylinder," Aeronaut. J. 78, 36 (1974).

${ }^{17}$ M. Raffel, C. Willert, and J. Kompenhans, Particle Image Velocimetry: A Practical Guide (Springer-Verlag, Berlin, 1998).

${ }^{18}$ K. D. Jensen, "Flow measurements," J. Braz. Soc. Mech. Sci. 26, 400 (2004).

${ }^{19}$ R. J. Moffat, "Contributions to the theory of single-sample uncertainty analysis," ASME J. Fluids Eng. 104, 250 (1982).

${ }^{20} \mathrm{~T}$. Nishino, "An investigation of flow around a circular cylinder in ground effect," M.Phil./Ph.D. transfer thesis, School of Engineering Sciences, University of Southampton, UK (2006).

${ }^{21}$ M. M. Zdravkovich, V. P. Brand, G. Mathew, and A. Weston, "Flow past short circular cylinders with two free ends," J. Fluid Mech. 203, 557 (1989).

${ }^{22}$ J. Zhou, R. J. Adrian, S. Balachandar, and T. M. Kendall, "Mechanisms for generating coherent packets of hairpin vortices in channel flow," J. Fluid Mech. 387, 353 (1999).

${ }^{23}$ P. W. Bearman and D. M. Trueman, "An investigation of the flow around rectangular cylinders,” Aeronaut. Q. 23, 229 (1972).

${ }^{24}$ S. Taniguchi and K. Miyakoshi, "Fluctuating fluid forces acting on a circular cylinder and interference with a plane wall," Exp. Fluids 9, 197 (1990).

${ }^{25}$ A. Roshko, "On the wake and drag of bluff bodies," J. Aeronaut. Sci. 22, 124 (1955).

${ }^{26}$ H. Oertel, Jr., "Wakes behind blunt bodies," Annu. Rev. Fluid Mech. 22, 539 (1990).

${ }^{27}$ P. Huerre and P. A. Monkewitz, "Local and global instabilities in spatially developing flows," Annu. Rev. Fluid Mech. 22, 473 (1990).

${ }^{28}$ R. J. Martinuzzi, S. C. C. Bailey, and G. A. Kopp, "Influence of wall proximity on vortex shedding from a square cylinder," Exp. Fluids 34, 585 (2003)

${ }^{29}$ X. Zhang, S. Mahon, M. Van Den-Berg, and C. Williams, "Aerodynamics of a half-cylinder in ground effect," in Engineering Turbulence Modelling and Experiments 6 (Proceedings of the ERCOFTAC International Symposium on Engineering Turbulence Modelling and Measurements-ETMM6), edited by W. Rodi and M. Mulas (Elsevier, Amsterdam, 2005), pp. 461470 . 